\title{
Analysis of SALT Fabry-Pérot Medium Resolution Data
}

\author{
Tapsoba, W. B. ${ }^{* 1,2}$, Williams, T. B. ${ }^{1,2}$, Carignan, C. ${ }^{1}$, Lucero, D. M. ${ }^{1}$ \\ ${ }^{1}$ Department of Astronomy, University of Cape Town, Private Bag X3, Rondebosch 7701, South \\ Africa \\ ${ }^{2}$ South African Astronomical Observatory, PO Box 9, 7935, Cape Town, South Africa ${ }^{\dagger}$ \\ E-mail: twblaiseler@gmail.com
}

\begin{abstract}
We observed the three galaxies NGC 7361, NGC 7424 and NGC 7793, using the Southern African Large Telescope (SALT) Fabry-Pérot interferometer in its medium resolution mode. These galaxies were selected from the MHONGOOSE (MeerKAT HI Observations of Nearby Galactic Objects: Observing Southern Emitters) sample. Measurements of the $\mathrm{H} \alpha$ emission line were used to produce velocity maps with spatial resolution of 2 arc-seconds and accuracy ranging from 1 $\mathrm{km} \mathrm{s}^{-1}$ to $10 \mathrm{~km} \mathrm{~s}^{-1}$. These maps were analyzed using the DiskFit and ROTCUR software packages to compute kinematic models and rotation curves.
\end{abstract}

SALT Science Conference 2015 -SSC2015-

1-5 June, 2015

Stellenbosch Institute of Advanced Study, South Africa

\footnotetext{
* Speaker.

$\dagger$ This research has made use of the NASA/IPAC Extragalactic Database (NED) which is operated by the Jet Propulsion Laboratory, California Institute of Technology, under contract with the National Aeronautics and Space Administration.
} 


\section{Introduction}

SALT (Southern African Large Telescope) Fabry-Pérot (FP) data have been obtained as part of the MHONGOOSE ancilliary survey. We observed three galaxies from this survey: NGC 7361, NGC 7424, NGC 7793, using the FP medium resolution (MR) mode of the RSS (Robert Stobie Spectrograph) on SALT. The goal is to get optical high spatial resolution ( $\sim 2 \operatorname{arcsec})$ data that will be used for a kinematic analysis in this study, [1,2,3] have shown that galaxy mass models are mainly constrained by the rising inner part of the rotation curve. This is better sampled by the present FP $\mathrm{H} \alpha$ data then by $21 \mathrm{~cm}$ HI measurements.

Numerous galaxies have been observed by others using FP techniques and the $\mathrm{H} \alpha$ line: including the GHASP sample [4, 14], Sculptor group galaxies [11, 16, 17], the Virgo sample [8, 9], the $\mathrm{BH} \alpha$ bar barred spiral galaxies sample [15] and the SINGS sample [10, 11].

This paper is presented as follows. In Section 2 and 3, we discuss respectively the data acquisition and reduction, respectively. In Section 4 the observed kinematic maps, the kinematic models using the two software packages DiskFit and ROTCUR and the rotation curve of each galaxy is presented.

\section{Data acquisition}

Three southern spiral galaxies selected from the MHONGOOSE sample were observed with the MR etalon of the FP imaging spectrograph. Our spectroscopic data covered the full velocity range of each galaxy with sample step of $\sim 90 \mathrm{~km} \mathrm{~s}^{-1}(2 \AA)$, properly sampling the instrumental velocity resolution. Table 1 gives the details of the observations.

Table 1: Basic data for NGC 7361, NGC 7424 \& NGC 7793.

\begin{tabular}{lcccccccc}
\hline \hline $\begin{array}{l}\text { Galaxies } \\
\text { NGC }\end{array}$ & $\begin{array}{c}\text { Type } \\
\text { RC3 }\end{array}$ & $\begin{array}{c}\alpha(\mathbf{J 2 0 0 0}) \\
(\mathrm{h} \mathrm{m} \mathrm{s})\end{array}$ & $\begin{array}{c}\delta(\mathbf{J 2 0 0 0}) \\
\left({ }^{\circ} \quad \prime^{\prime \prime}\right)\end{array}$ & $\begin{array}{c}\mathrm{V}_{\text {sys }} \\
\left(\mathrm{km} \mathrm{s}^{-1}\right)\end{array}$ & $\begin{array}{c}\text { Distance } \\
(\mathrm{Mpc})\end{array}$ & $\begin{array}{c}\text { Date of } \\
\text { observations }\end{array}$ & $\begin{array}{c}\text { Total exposure time } \\
(\mathrm{sec})\end{array}$ & 00 \\
\hline $\mathbf{7 3 6 1}$ & $\mathrm{S}(\mathrm{r}) \mathrm{c}$ & 224217.9 & -300328 & $1249 \pm 3^{a}$ & $15.9^{b}$ & $2012-08-23$ & 2200 \\
$\mathbf{7 4 2 4}$ & $\mathrm{SAB}(\mathrm{rs}) \mathrm{cd}$ & 225718.4 & -410414 & $939 \pm 2^{a}$ & $13.1^{c}$ & $2012-09-04$ & 2200 \\
$\mathbf{7 7 9 3}$ & $\mathrm{SA}(\mathrm{s}) \mathrm{d}$ & 235749.8 & -323528 & $230 \pm 4^{d}$ & $4.0^{c}$ & $2012-09-24$ & 2035 \\
\hline
\end{tabular}

${ }^{a}[18]$

${ }^{b}[24]$

${ }^{c}[21]$

${ }^{d}[13]$

\section{Data reduction}

The data reduction procedure is fully described by [27]. The key steps of the reduction are:

- Standard reduction scheme for CCD images using IRAF: cosmic ray removal using L.A.Cosmic [28]; flat fielding; flux calibration;

- The FP key reduction steps: 


\section{Wavelength calibration:}

- Night-Sky line identifications. In order to identify the night-sky lines present in each image we use Equation 3.1 to calculate the wavelength of the spectral feature and then use spectral information from [23] to identify the lines.

- Calibration parameter determination. We used the identified night-sky lines to determine the wavelength calibration, expressed in:

$$
\lambda=\frac{\mathrm{A}+\mathrm{BZ}+\mathrm{Et}}{\sqrt{1+\left(\frac{\mathrm{r}}{\mathrm{f}_{\mathrm{cam}}}\right)^{2}}}
$$

Where $\mathrm{Z}$ is the FP control parameter, $\mathrm{t}$ the time of observation; and the calibration constants are $\mathrm{A}, \mathrm{B}, \mathrm{E}$, and $f_{\text {cam }}$.

2. Sky-line removal. Once the night-sky emission lines have been used for wavelength calibration, they must be removed from the images since they often overlap the image of the galaxy. The night sky line subtraction is done by fitting a Lorenzian profile to the sky lines in the spectrum of each frame.

3. Ghost removal. The main ghost arises from reflection between the CCD detector and the Fabry-Pérot etalon. The ghost image is mirror-symmetric about the optical axis and in focus.

4. Seeing normalisation. We use the IRAF task PSFMATCH to convolve all the images to the same seeing.

\section{Results}

\subsection{Observed kinematics maps}

To compute the observed kinematic maps, a spectrum at each pixel is fitted with a Voigt function ([21]) to give :

- the line center $\lambda_{\text {peak }}$ and its uncertainty $\Delta \lambda_{\text {peak }}$,

- the continuum strength $\mathrm{I}_{0}$ and its uncertainty $\Delta \mathrm{I}_{0}$.

- the intensity I or line strength and its uncertainty $\Delta \mathrm{I}$.

The final velocity maps, corrected to the heliocentric frame, are shown in Figure 1.

\subsection{Kinematic analysis with DiskFit and ROTCUR}

The kinematic analysis was carried out using the two software packages DiskFit (method described by [25]) and ROTCUR (method taken from [16]). As seen below, the two packages produce results that agree within the estimated uncertainties. 

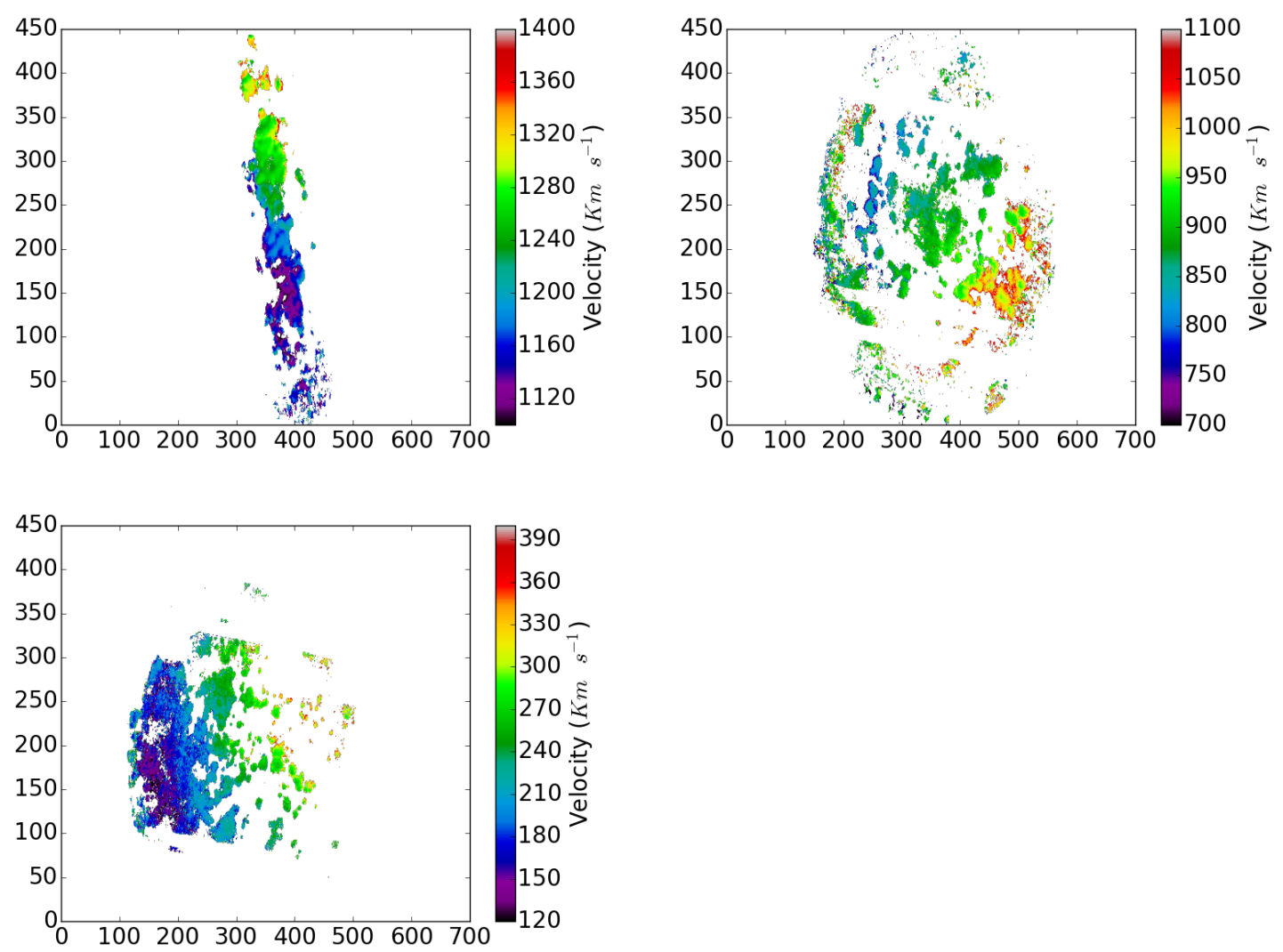

Figure 1: Top left: NGC 7361 velocity map; top right: NGC 7424 velocity map; bottom left: NGC 7793 velocity map. The $\mathrm{x}$ - and $\mathrm{y}$-axis are in pixel with $0.506 \mathrm{arcsec} / \mathrm{pixel}$.

\subsection{Best fitted parameters determination procedure}

The following description of DiskFit is taken from [27]. To determine the observed velocity field parameters $\phi, i, \varepsilon, x_{c}$ and $y_{c}$ the following steps are used for all the three galaxies using both DiskFit and ROTCUR :

1. Computation of the ellipticity using a SPITZER Space Telescope ${ }^{(1)} 3.6 \mu \mathrm{m}$ mid-infrared image. We used the $3.6 \mu \mathrm{m}$ mid-infrared image because it is more representative of the old disk population, which accounts for most of the mass. For the same reason, the bulge, which has mainly an old population, is very well traced. We set the center of the galaxy to the brightest pixel and we use the IRAF task $\operatorname{ELLIPSE}^{(2)}$ to fit elliptical isophotes to the infrared image. This gives the ellipticities $\varepsilon_{i}$ for a set of annuli starting from a radius that does not include the galaxy bulge. From that we compute the ellipticity of the galaxy as the mean ellipticity.

2. We calculate the inclination $i$ from the ellipticity by :

$$
i=\cos ^{-1}(1-\varepsilon)
$$

${ }^{1}$ SPITZER Space Telescope (SST) is an mid-infrared space observatory launched in 2003.

${ }^{2}$ The IRAF task GEOMPAR sets the geometric parameters of the galaxy for the 'ellipse' task 
3. The center $\left(x_{c}, y_{c}\right)$ of the observed galaxy set to the pixel with highest intensity within the nucleus.

4. We keep the ellipticity $\varepsilon$, the inclination $i$ and the center of our galaxy images fixed for both ROTCUR and DiskFit and we fit :

- first for the systemic velocity $V_{s y s}$

- and we use this fitted value of the $V_{\text {sys }}$ to compute the position angle $\phi$.

5. We held all the parameters $V_{s y s}$, the center $\left(x_{c}, y_{c}\right)$, the inclination $i$ and $\phi$ fixed and fit for the rotation velocity $V_{c}$.

6. We estimate the correction $\phi_{\text {rot }}$ for all the galaxy by rotating our Fabry-Pérot image with respect to the SPITZER Space Telescope image using the data visualization application SAOImage DS9 $9^{(3)}$.

The angular size, the position angle P.A and the inclination are from ESO-LV "Quick Blue": IIa-O band.

\subsubsection{NGC 7361 kinematic analysis}

The kinematic fits for NGC 7361 are given in Table 2 and the top left panel of Figure 2. [20] found a rotational velocity $v_{\text {rot }}=103 \mathrm{~km} \mathrm{~s}^{-1}$ from an HI profile of NGC 7361 obtained with the 64 m Parkes radio telescope, CSIRO. Mathewson et al. (1992) also present an optical rotation curve from long-slit major axis spectra, shown in blue in the top left panel of Figure 2. Our measurements are in reasonable agreement. Our determination of the position angle (P.A.) and the inclination (i) agree well with values from the literature. Our systemic velocity determination differs significantly from previous measurements listed in Table 2, we do not understand this discrepancy. The rotation curves are rising linearly from the center up to 34 arcsecs (constant density in the inner part) and then flatten until the end of our measurement (density falling as 1/r, with $r$ the radial distance).

Table 2: Comparison with literature. Case of NGC 7361

\begin{tabular}{lccc}
\hline \hline References & $\begin{array}{c}\mathrm{V}_{\text {sys }} \\
\left(\mathrm{kms}^{-1}\right)\end{array}$ & $\begin{array}{c}\text { P.A. } \\
\left({ }^{\circ}\right)\end{array}$ & $\begin{array}{c}i \\
\left({ }^{\circ}\right)\end{array}$ \\
\hline DiskFit & 1192.0 & 4.5 & 76.1 \\
ROTCUR & 1192.9 & 3.0 & 76.1 \\
{$[18]$} & 1249 & - & - \\
{$[20]$} & 1243 & 4 & 87 \\
{$[19]$} & - & 3 & 76 \\
\hline
\end{tabular}

\footnotetext{
${ }^{3}$ SAOImage DS9 is data visualization application and astronomical imaging. http://ds9.si.edu/site/ Home.html.
} 


\subsubsection{NGC 7424 kinematic analysis}

Our results for NGC 7424 are present in Table 3 and the top right panel of Figure 2. [5] estimated a maximum rotation velocity of NGC 7424 to be $\sim 150 \mathrm{~km} \mathrm{~s}^{-1}$ and [26] found a value $\sim$ $146 \mathrm{~km} \mathrm{~s}^{-1}$. Our observed maximum rotation velocity is about $\sim 150 \mathrm{~km} \mathrm{~s}^{-1}$, which is in agreement with the two previous values. Our position angle (P.A.), inclination (i) and systemic velocity $V_{s y s}$ determination are in decent agreement with the values from the literature. The rotation curves are rising linearly from the inner part up to the end of our measurement, which implies a constant mass density through the fitted radius.

Table 3: Comparison with literature. Case of NGC 7424.

\begin{tabular}{lccc}
\hline \hline References & $\begin{array}{c}\mathrm{V}_{\text {sys }} \\
\left(\mathrm{km} \mathrm{s}^{-1}\right)\end{array}$ & $\begin{array}{c}\text { P.A. } \\
\left({ }^{\circ}\right)\end{array}$ & $\begin{array}{c}i \\
\left({ }^{\circ}\right)\end{array}$ \\
\hline DiskFit & 914.6 & 133.8 & 58.8 \\
ROTCUR & 915.4 & 131.6 & 58.8 \\
{$[18]$} & 939 & - & - \\
{$[19]$} & - & 125 & 49 \\
\hline
\end{tabular}

\subsubsection{NGC 7793 kinematic analysis}

Our results for NGC 7424 are present in Table 3 and the bottom left panel of Figure 2. [20] found a rotational velocity $v_{\text {rot }}=120 \mathrm{~km} \mathrm{~s}^{-1}$ through an HI profile of NGC 7793 obtained with the $64 \mathrm{~m}$ Parkes radio telescope. [7] HI profile (VLA Observations) gives an estimated maximum rotational velocity of about $110 \mathrm{~km} \mathrm{~s}^{-1}$. These maximum rotation velocities from the literature are in excellent agreement with our measurement from the bottom panel of Figure 2 which is about $110 \mathrm{~km} \mathrm{~s}^{-1}$.

To compare our results with those of [11], we plotted the data they obtained together with our observed data up to a maximum radius of 180 arcsecs (our observed data limit). We notice that our rotation curve and the one of [12] agree well up to a radius of 40 arcsecs within the inner portion of the galaxy, but disagree at at larger radii. Our rotation curve agrees well with that of [7] only to $r \sim 80^{\prime \prime}$. Significant disagreement from $80^{\prime \prime}$ to $130^{\prime \prime}$ and beyond $160^{\prime \prime}$. Table 4 shows that the position angle (P.A.), the inclination (i) and the systemic velocity $V_{\text {sys }}$ are in good agreement with the values from the literature. As for NGC 7361, the sharp dip and recovery in the rotation curve is unphysical and suggest that some error may occur during analysis.

Table 4: Comparison with literature. Case of NGC 7793.

\begin{tabular}{lccc}
\hline \hline References & $\begin{array}{c}\mathrm{V}_{\text {sys }} \\
\left(\mathrm{km} \mathrm{s}^{-1}\right)\end{array}$ & $\begin{array}{c}\text { P.A. } \\
\left({ }^{\circ}\right)\end{array}$ & $\begin{array}{c}i \\
\left({ }^{\circ}\right)\end{array}$ \\
\hline DiskFit & 223.8 & 288.5 & 50.9 \\
ROTCUR & 223.5 & 288.8 & 50.9 \\
{$[11]$} & 230 & 286 & 47 \\
{$[20]$} & 226 & - & 54 \\
\hline
\end{tabular}



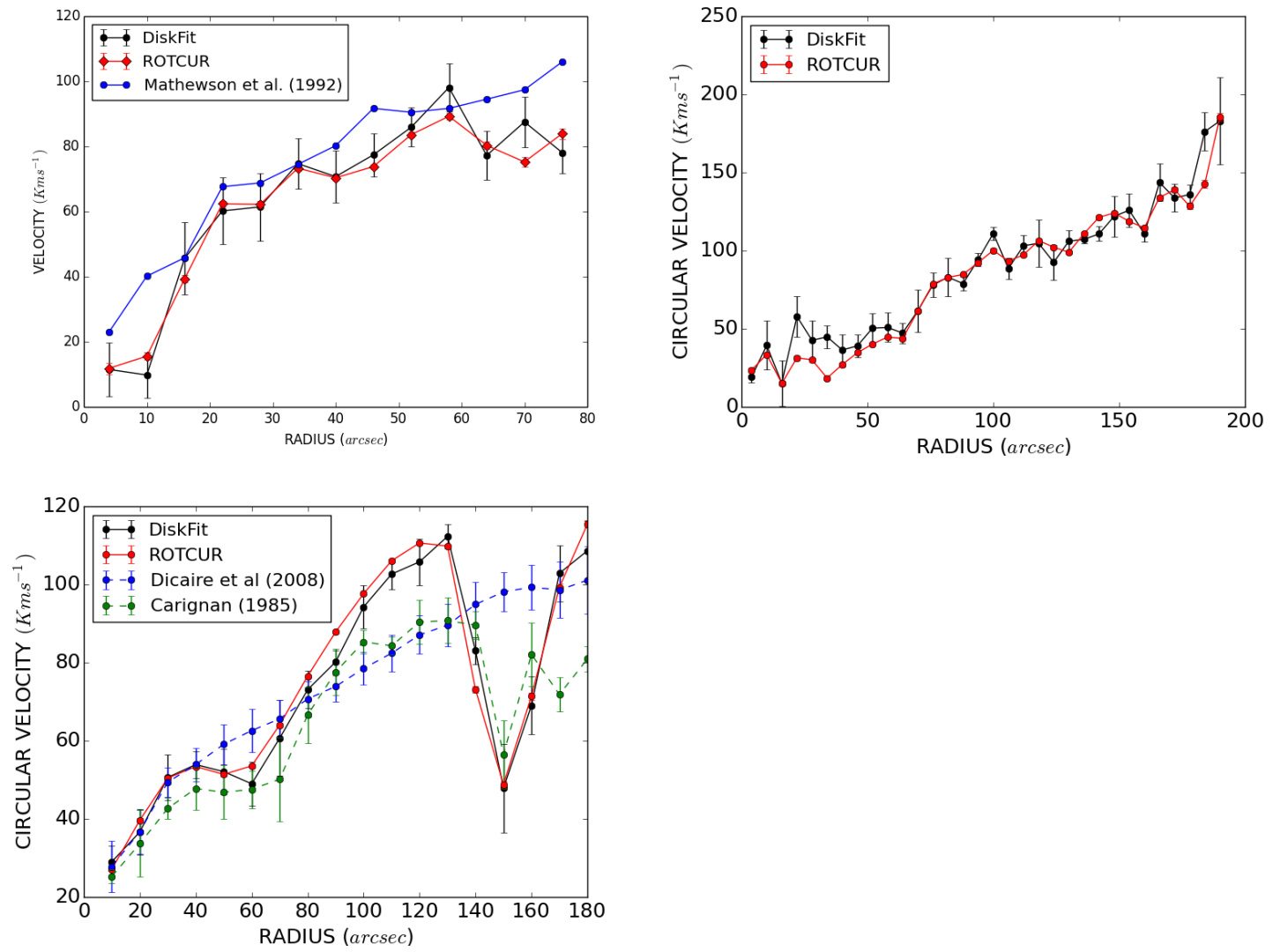

Figure 2: Rotation curves of: NGC 7361 top left; NGC 7424 top right; NGC 7793 bottom left

\section{Conclusion}

We used the SALT RSS Fabry-Pérot interferometer in its medium resolution to observe the three spiral galaxies: NGC 7361, NGC 7424 and NGC 7793 . We produced velocity maps with estimated uncertainties ranging from $1 \mathrm{~km} \mathrm{~s}^{-1}$ to $10 \mathrm{~km} \mathrm{~s}^{-1}$. We used the software packages DiskFit and ROTCUR to produce both kinematic maps and rotation curves for each galaxy. The observed velocity parameters obtained after analysis were in agreement not only with both methods (DiskFit \& ROTCUR) but more importantly with the results from the literature.

\section{References}

[1] Amram, P., Balkowski, C., Boulesteix, J., Cayatte, V., Marcelin, M., \& Sullivan, III, W. T. 1996, A\&A, 310, 737

[2] Amram, P., Boulesteix, J., Marcelin, M., Balkowski, C., Cayatte, V., \& Sullivan, III, W. T. 1995, A\&AS, 113, 35

[3] Amram, P., Marcelin, M., Balkowski, C., Cayatte, V., Sullivan, III, W. T., \& Le Coarer, E. 1994, A\&AS, 103, 5

[4] Amram, P. \& Garrido, O. 2002, in Astronomical Society of the Pacific Conference Series, Vol. 282, Galaxies: the Third Dimension, ed. M. Rosada, L. Binette, \& L. Arias, 103 
[5] Bajaja, E. \& Martin, M. C. 1985, AJ, 90, 1783

[6] Begeman, K. G. 1989, A\&A, 223, 47

[7] Carignan, C. \& Puche, D. 1990, AJ, 100, 394

[8] Chemin, L., Balkowski, C., Cayatte, V., Carignan, C., Amram, P., Garrido, O., Hernandez, O., Marcelin, M., Adami, C., Boselli, A., \& Boulesteix, J. 2006, MNRAS, 366, 812

[9] Chemin, L., Cayatte, V., Balkowski, C., Amram, P., Carignan, C., Boselli, A., Adami, C., Marcelin, M., Garrido, O., Hernandez, O., \& Boulesteix, J. 2005, A\&A, 436, 469

[10] Daigle, O., Carignan, C., Amram, P., Hernandez, O., Chemin, L., Balkowski, C., \& Kenni- cutt, R. 2006, MNRAS, 367, 469

[11] Dicaire, I., Carignan, C., Amram, P., Marcelin, M., Hlavacek-Larrondo, J., de Denus- Baillargeon, M.-M., Daigle, O., \& Hernandez, O. 2008a, AJ, 135, 2038

[12] Dicaire, I., Carignan, C., Amram, P., Hernandez, O., Chemin, L., Daigle, O., de Denus- Baillargeon, M.-M., Balkowski, C., Boselli, A., Fathi, K., \& Kennicutt, R. C. 2008b, MNRAS, 385, 553

[13] de Vaucouleurs, G., de Vaucouleurs, A., Corwin, Jr., H. G., Buta, R. J., Paturel, G., \& Fouque, P. $1991, \mathrm{~S} \& \mathrm{~T}, 82,621$

[14] Epinat, B., Amram, P., \& Marcelin, M. 2008, MNRAS, 390, 466

[15] Hernandez, O., Carignan, C., Amram, P., Chemin, L., \& Daigle, O. 2005, MNRAS, 360, 1201

[16] Hlavacek-Larrondo, J., Carignan, C., Daigle, O., de Denus-Baillargeon, M.-M., Marcelin, M., Epinat, B., \& Hernandez, O. 2011a, MNRAS, 411, 71

[17] Hlavacek-Larrondo, J., Marcelin, M., Epinat, B., Carignan, C., de Denus-Baillargeon, M.- M., Daigle, O., \& Hernandez, O. 2011b, MNRAS, 416, 509

[18] Koribalski, B. S., \& al, 2004, AJ, 128, 16

[19] Lauberts, A. \& Valentijn, E. A. 1989, The Messenger, 56, 31

[20] Mathewson, D. S., Ford, V. L., \& Buchhorn, M. 1992, ApJS, 81, 413

[21] Menéndez-Delmestre, K., Sheth, K., Schinnerer, E., Jarrett, T. H., \& Scoville, N. Z. 2007, ApJ, 657, 790

[22] Mitchell, C. J., Williams, T. B., Spekkens, K., Lee-Waddell, K., Kuzio de Naray, R., \& Sellwood, J. A. 2014

[23] Osterbrock, D. E., Fulbright, J. P., Martel, A. R., Keane, M. J., Trager, S. C., \& Basri, G. 1996, PASP, 108,277

[24] Rossa, J. \& Dettmar, R.-J. 2003, A\&A, 406, 505

[25] Spekkens, K. \& Sellwood, J. A. 2007, ApJ, 664, 204

[26] Staveley-Smith, L. \& Davies, R. D. 1988, MNRAS, 231, 833

[27] Tapsoba, W., B., Williams, T., Carignan, C., Lucero, D., M., Master thesis, University of Cape Town, June 2015

[28] van Dokkum, P. G. 2001, PASP, 113, 1420 